Instituto Internacional de Investigación y Desarrollo Tecnológico Educativo INDTEC, C.A.

DOI: https://doi.org/10.29394/Scientific.issn.2542-2987.2018.3.7.18.354-373

OAI-PMH: http://www.indteca.com/ojs/index.php/Revista Scientific/oai

\title{
Gestión Docente en el Contexto de la Educación Primaria Venezolana
}

\author{
Autora: Marilin Pastora Arrieche Cordero \\ Universidad Fermín Toro, UFT \\ marilinarrieche@hotmail.com
}

Lara, Venezuela

\section{Resumen}

Uno de los aspectos fundamentales de un país es la formación de sus estudiantes por lo cual el tema abordado en este ensayo es la Gestión Docente de Educación Primaria en el contexto Educativo Venezolano. Tomando en consideración que la educación de los niños, niñas y adolescente va a depender del desenvolvimiento del educador, donde debe estar actualizado sobre los avances del conocimiento, sobre todo en la parte pedagógica y como sujeto en interacción con sus discípulos, debe conocerlos y centrar su acción en el amor y el diálogo, por lo tanto en esa plenitud de gerenciar la praxis educativa el maestro de la vida quien busca la excelencia de sus educandos, para así llevar a cabo esa fuente de conocimiento de esa realidad, donde el profesor y el aprendiz, conjugando así los saberes y experiencias, promuevan ese cambio de mentalidad que transforme al sujeto que imparte cultura, en un diálogo profundo para alcanzar una educación para la vida, el logro, y el cambio. Un sistema educativo venezolano que este a la altura de las grandes transformaciones y cambios mundiales, para así ese paradigma de innovación sea para instaurar y construir la justicia, la equidad y la paz. Una instrucción que lleve ideas que formen al ciudadano venezolano, para el progreso y el desarrollo del pueblo, en el campo educativo, socioeconómico, cultural y religioso.

Palabras clave: gestión; docente; educación. 


\title{
Teaching Management in the Context of Venezuelan Primary Education
}

\begin{abstract}
One of the fundamental aspects of a country is the training of its students, so the topic addressed in this essay is the Teaching Management of Primary Education in the Venezuelan Educational context. Taking into consideration that the education of children and adolescents will depend on the development of the educator, where he must be updated on the advances of knowledge, especially in the pedagogical part and as subject in interaction with his disciples, he must know them and center his action in love and dialogue, therefore in that plenitude of managing the educational praxis the teacher of life who seeks the excellence of his students, in order to carry out that source of knowledge of that reality, where the teacher and the learner, thus combining knowledge and experiences, promote that change of mentality that transforms the subject that imparts culture, in a deep dialogue to achieve an education for life, achievement, and change. A Venezuelan educational system that lives up to the great transformations and global changes, so that paradigm of innovation is to establish and build justice, equity and peace. An instruction that carries ideas that form the Venezuelan citizen, for the progress and development of the people, in the educational, socioeconomic, cultural and religious field.
\end{abstract}

Keywords: management; teaching; education. 


\section{Introducción}

El hombre en la búsqueda de su autorrealización va reflexionando como es su interioridad, utilizando la filosofía clásica griega conoce a ti mismo de Sócrates, esto concientiza en que se ha de buscar en el alma, la fuente de una construcción personal, hacia una fuente rica de pensamiento creativo y crítico entre una postura como educador en la construcción de una verdad de una teoría y una praxis de un Sistema Educativo Venezolano que, connote, vivifique y actualice un razonamiento para la vida, para una escuela de integridad de conocimiento holístico.

En primer lugar, la educación tiene que estar centrada en el hombre, que es el sujeto de acción y de cambio transformador de la sociedad, de esta manera se puede observar, que corresponde la responsabilidad del maestro venezolano, en formar al estudiante con pensamiento crítico, heurístico, creativo y artístico. Todo ser humano tiene que estar con un motivo y en un afán de hacer las cosas en educación, en caridad, en eficiencia y en crear un logro motivacional, en el campo personal, en la búsqueda del desarrollo de la personalidad para así fomentar el ser humano, apto para la vida democrática.

La educación debe formar un individuo integro, actualizado, capaz de enfrentarse a los cambios y de resolver efectivamente los problemas actuales. Para ello es necesario una estrecha relación entre las instituciones educativas y su entorno político, social y económico, en donde debe actualizarse diseños curriculares adecuados a las necesidades del individuo y la sociedad, disponer de estrategias metodológicas adaptadas a los últimos avances tecnológicos.

Así mismo en el informe, la Educación Encierra un tesoro, elaborado por una comisión de la UNESCO (1996), destaca una serie de tensiones que están en el centro de la problemática de la educación del hombre del siglo XXI.

La tensión entre el extraordinario desarrollo de los conocimientos y las capacidades de asimilación del ser humano. La comisión no resistió a la tentación de añadir nuevas disciplinas como el conocimiento de sí mismo y los 
medios de mantener la salud física y psicológica, o el aprendizaje para conocer mejor el medio ambiente natural y preservarlo. $Y$ sin embargo los programas escolares cada vez están más recargados. Por tanto, será necesario escoger en una clara estrategia de reforma, pero a condición de preservar los elementos esenciales de una educación básica que enseñe a vivir mejor mediante el conocimiento, la experimentación formación de una cultura personal. (pág. 12).

Esta comisión plantea una educación para la vida, formada en valores, tomando en consideración el ser, hacer, convivir y conocer, para la formación de un individuo integral. Conscientes de la época tecnológica, informatizada, globalizada en que vivimos, la educación debe redefinirse en cuanto sus fines, contenidos estrategias y roles del docente, estos deben estar orientados a la formación del hombre para la vida, es decir para la reflexión, el trabajo y la felicidad.

De acuerdo con lo expuesto anteriormente se desarrolla esta investigación, para interpretar la gestión docente en Educación Primaria en el Contexto Venezolano, con la finalidad de detectar el desenvolvimiento del mismo y de esta manera contribuir a dar aportes para el desarrollo de un sistema Educativo, acorde a la realidad del país, para responder a una demanda cada vez mayor que exige calidad y equidad.

En la educación primaria abarcando todas las herramientas básicas del aprendizaje tanto en lo ético y cultural, lo científico y tecnológico, económico, social. Desde esta perspectiva, la educación se constituye en el elemento indispensable para la promoción y formación integral del ser humano y de estar consustanciado con los cambios sociales que sean el resultado de un proceso de aprendizaje.

En este sentido Botero (2009a),

La gestión educativa "busca aplicar los principios generales de la gestión que han estado presentes en la teoría de la administración, al campo específico de la educación. El objeto 
de la gestión educativa como disciplina, es el estudio de la organización del trabajo en el campo de la educación, por lo tanto, está influenciada por teorías de la administración, pero además existen otras disciplinas que han permitido enriquecer el análisis como son: la administración, la filosofía, las ciencias sociales, la psicología, la sociología y la antropología”. (pág. 2).

Sin duda alguna la gestión es una palabra que se ha venido usando en la cultura docente muy intensamente en la última década, pero casi nunca nos detuvimos a preguntar por su procedencia y significado real. Este término, viene en el mismo paquete de cambios en educación y pocos percibieron su incidencia. Como otros términos prestados de otras ciencias e introducidos en la cultura educativa como moda, fueron acogidos de manera mecánica por los docentes y autoridades para darle cierta modernidad a su discurso.

Según, Vargas (2008), la gestión educativa "es vista como un conjunto de procesos teóricos-prácticos integrados horizontal y verticalmente dentro del sistema educativo, para cumplir los mandatos formales, en donde la gestión debe ser entendida como una forma de comprender y conducir la organización escolar". (pág. 68). Citado por Ramírez (2012). Lo cual significa que la gestión sería un conjunto de procedimientos para encaminar la administración académica. La educación se inscribe en un marco de principios éticos y valores que caracterizan el ejercicio de la función docente. En consecuencia, el maestro es ético cuando actúa con responsabilidad en un contexto institucional, teniendo en cuenta que comparte con sus estudiantes tanto su ser como su hacer y el conocimiento que posee.

El docente debe enfrentarse a grandes desafíos como desarrollar la capacidad de adaptación, toma de decisiones en un argumento de alta incertidumbre. En este contexto la formación permanente se constituye en un elemento fundamental para asumir con responsabilidad y criterio la formación de los alumnos, para ello requiere de procesos de actualización permanente. Por otro lado, Carrasco, (2002), define la gestión educativa "como un aspecto 
fundamental de la educación, juega un papel importante en la conducción y realización de las actividades, que van a conducir al logro de las metas y objetivos previstos en el sistema educativo", (pág. 16).

Es preciso considerar que el logro de un aprendizaje de calidad, es un reto al que se enfrentan los sistemas educativos en el mundo, lo cual exige hacer énfasis en la formación de los futuros científicos, profesionales, tecnólogos y técnicos necesarios a incorporar a la esfera productiva para el desarrollo. La educación con calidad busca formar ciudadanos capaces de comprender las complejas interrelaciones existentes entre ciencia, tecnología, ámbitos sociales, económicos, políticos y culturales con la finalidad que, en el futuro y cualquiera que sea su esfera de actividad, cuenten con los instrumentos imprescindibles para participar en la toma de decisiones que contribuya a construir una sociedad democrática.

\section{Desarrollo}

El docente origina cambios y transformaciones favorables, en relación con el logro de las líneas estratégicas establecidas en el contexto social y las políticas educativas. En este sentido, para que un docente lleve a cabo su función de manera efectiva en la organización escolar, es de vital importancia que ejerza su función acorde con su crecimiento personal y profesional. Sus acciones representan un compromiso fundamental donde se coordinan esfuerzos para formar seres humanos en las mejores condiciones; lo cual implica que es indispensable ejecutar acciones que conformen un equipo de trabajo para responder a los requerimientos de la organización educativa.

Debido a esto, es primordial que cualquier sistema educativo se aboque a mejorar, puesto que su objetivo central es lograr un justo equilibrio, además de una adecuada optimización de la eficiencia y eficacia de la educación, contribuyendo de esta manera a la consolidación del mejoramiento continuo como parte inherente a la búsqueda de la calidad educativa. Asimismo, existe 
la necesidad de buscar nuevas articulaciones, la cual va referida al grado de dominio de informaciones y destrezas que debe poseer el docente, teniendo presente que esta articulación implica un proceso de nuevos consensos, compromisos y responsabilidades.

Estos cambios se están generando a nivel de la educación, en todos los ámbitos son retos para el docente, quien debe lograr una educación de calidad, y preocuparse por la actualización para enfrentar los desafíos que se presentan en la sociedad con una economía globalizada y la competencia internacional de los diferentes espacios del quehacer educativo. Desde esta perspectiva Montenegro (2003), define el desempeño docente como "el desempeño de sus funciones; ésta se halla determinado por actores asociados al propio docente, al estudiante y al entorno" (pág. 18).

Ante tales consideraciones, se reflexiona sobre los diferentes actores que determinan la labor docente los cuales son: su formación profesional, sus condiciones de salud y el grado de motivación y compromiso por su labor. La parte profesional abarca la planificación organizada y la evaluación constante, por lo tanto, mientras mayor calidad tenga de preparación, la eficiencia será en un grado más elevado. Asimismo, un docente que goce de buena salud tendrá mayor posibilidad de ejercer sus funciones a cabalidad, con satisfacción; y la motivación va a depender del incentivo que tenga para llevar a cabo la labor en la cual se desenvuelve.

Por otro lado, el docente es considerado por Díaz y Hernández (2001), "como una persona encargada de conducir el proceso educativo" (pág. 23). De manera que, es el profesor el responsable de la gestión educativa, para lo cual debe responder a funciones específicas, tomando en consideración que debe cumplir distintos roles en la práctica del ejercicio pedagógico dichos roles son fundamentales a la hora de programar las competencias eficaces en la enseñanza.

La Educación ha sido a nivel mundial un tema central por el cual se han 
formulado diferentes estudios, sobre todo tomado en consideración la inclusión de los ciudadanos al sistema Educativo en donde todo el mundo tenga acceso al sistema Educativo. En el año 2000 se formularon 6 objetivos para el 2015 y diferentes procedimientos para ejecutarlos, esto con la representación de 164 países, que se congregaron en el foro mundial sobre Educación de la misma manera se hizo público el informe de seguimiento de la EPT (Educación para Todos), donde se da a conocer el desempeño logrado. Igualmente se señalan las enseñanzas básicas que deberían configurar la agenda mundial de Educación a partir de ahora.

Uno de los más relevantes retos que se han creado en el marco del avance de la ciencia histórica en los últimos años, en Venezuela y América Latina, es indiscutiblemente, el estudio cada vez más profundo, sistemático, ordenado y metódico que se viene manifestando en el campo histórico de la Educación. Es a finales del siglo XVIII, en la Caracas colonial de 1794, que se localiza el primer documento crítico elaborado por un maestro acerca de su oficio y las condiciones en las que encontraban los establecimientos de primeras letras en nuestro país. Se trata del informe elaborado por Simón Rodríguez al Cabildo de Caracas con el título de "Estado actual de la Escuela y nuevos establecimientos de ella" de fecha 19 de mayo de 1794.

El sistema educativo se ha venido desarrollando en Venezuela de forma muy lenta, tiene mucho que ver con los cambios ocurridos en la época, el cual ha pasado por muchos acontecimientos históricos dependiendo del momento. El presidente Antonio Guzmán Blanco presidente en el año 1870, establece por decreto la instrucción pública, gratuita y obligatoria, en el año 1873 el sistema educativo solo atendía 3.744 alumnos, en 100 escuelas públicas.

Para el año 1936, la educación se convierte en unas de las prioridades del estado venezolano y el Ministerio cambia de nombre a Educación Nacional. Para el año 1948 se crea la ley orgánica de educación Nacional y se impulsa el proceso de alfabetización de adultos. Es entonces a principio de la década 
de los 60 , cuando la educación venezolana presenta una considerable extensión y un crecimiento notorio, en el cual tuvieron acceso todas las clases sociales del país.

Haciendo un breve recuento de cómo ha progresado la educación en los últimos años, se puede decir que han ingresado un gran número de estudiantes al sistema educativo, sin embargo, no se puede decir lo mismo con respecto a la calidad de alumnos que egresan. Las reformas educativas son hechas en los cuales el gobierno instaura los componentes, para encauzar las políticas de la enseñanza. Son el fruto de un desarrollo profundo, en el que intervienen elementos intrínsecos y superficiales a la autenticidad colectiva de una nación.

A finales del siglo $X X$, surgieron una serie de acontecimientos que describen esta etapa, entre las cuales se encuentran un conjunto de reformas que tienen un cúmulo de objetivos en común. Cabe señalar las siguientes: Igualdad en el derecho a la educación, examinar los contenidos que comprenden el currículo, así como también mejorar la calidad de la educación, entre otras.

Ya en materia de progreso como la globalización ha forzado al mundo a ejecutar transformaciones en sus elementos primordiales, tal es el caso de la Educación. Es por lo que diferentes países del mundo han fabricado sus propios planes teniendo en cuenta las condiciones de su entorno para afrontar las exigencias actuales y futuras a las que están siendo sometidas, como resultado del proceso global.

En este momento la política de reforma de estado en materia de educación, es fundamentalmente la masificación de la educación e innovación en metodología de enseñanza, puesto que el país está exigiendo un cambio en la manera de educar, ya que la demanda laboral va expandiéndose, es por ello que se han insertado en el país nuevos modelos de inserción escolar incluyendo la universitaria. Los proyectos que se están creando juegan un 
papel fundamental en la inclusión escolar en masas, puesto que el fin único es una educación globalizadora donde podamos crecer todos los ciudadanos y ciudadanas del país sin exclusión y en plena igualdad de condiciones.

Hoy, por hoy se está generando una transformación en la docencia, en el nuevo marco legal de las Orientaciones Pedagógicas 2015-2016, en donde especifica que La Atención Educativa Integral: "garantiza la atención de las y niñas, niños y adolescentes desde el primer grado hasta culminar el sexto grado, considerando la responsabilidad, la familias, escuela y comunidad" (pág. 32). Se puede decir que se recalca que la responsabilidad ya no es nada más del docente, la familia también juega un papel importante en la educación del estudiante, los deberes deben ser compartidos.

En estas orientaciones pedagógicas, está inmerso la parte de la tecnología con las portátiles canaimas a cargos de los contenidos adscritos a FUNDABIT, así como también la activación de los congresos pedagógicos, escolares, estadales, municipales y nacional, lo cual indica un nuevo enfoque curricular.

Igualmente en las Orientaciones Pedagógicas 2014-2015, orientación número 43 de la atención académica de la Educación Primaria, la cual explica, que los colectivos docentes propiciarán momentos diarios de lectura de 30 minutos (30) en los diferentes ambientes de aprendizaje, utilizando los libros de la colección bicentenario, la portátil Canaima y otros recursos (periódicos, folletos, trípticos, dípticos, cuentos, revistas, canciones, entre otros, se recomienda que a través del Centro de Recurso para el Aprendizaje, se promuevan ferias de Lecturas, encuentros de poesías y cuentos.

En la actualidad existe un currículo Nacional Bolivariano que especifica el subsistema de Educación Primaria (2007), "El subsistema de SEB garantiza la formación integral de los niños y las niñas desde los seis (6) hasta los doce (12) años o hasta su ingreso al subsistema siguiente". (pág. 25). La concentración, tanto en segmentos sociales nacionales, como en algunos 
países, junto con las grandes interrogantes que hoy se plantea la sociedad frente a la educación, que llevan a reflexionar acera de la identidad profesional del docente, sobre las formas y prácticas del desempeño profesional en el contexto de las actuales valoraciones y exigencias de la vida social, y sobre la nueva distribución de responsabilidades en la educación.

La humanidad actual ha sufrido transformaciones aceleradamente, debido a una serie de innovaciones en el campo tecnológico, científico y de telecomunicaciones influyendo en las actividades educativas realizadas en el salón de clase, para lo cual florece un nuevo tipo de alumno. Siguiendo este orden de ideas, Botero (2009b), menciona que "La gestión Educativa del siglo XXI, deberá trazar políticas de acción que cualifiquen a los trabajadores cuya preparación hoy puede estar por debajo de la tecnología empleada" (pág. 6). Por esta razón existe la necesidad de buscar nuevas articulaciones, la cual va referida al grado de dominio de informaciones y destrezas que debe poseer el docente, teniendo presente que esta articulación implica un proceso de nuevos consensos, compromisos y responsabilidades.

Que sea capaz de que una realidad en que se está inmerso en una sociedad globalizada y postmoderna, vincular una escala de valores en la formación e instrucción, para así crear la conciencia moral y social de un ciudadano vislumbrante, de incluir política educativa real, justa en una disposición de que esta patria Venezolana promulgue en el quehacer científico y tecnológico, con una conciencia filosófica, con un resaltamiento de la capacidad artística y un conocimiento de los valores transcendentales de la espiritualidad del Venezolano.

Así mismo, realzar una educación activa que promueva la sensatez e idoneidad del republicano, y que no esté divorciada de la realidad determinante, de la estructura económica nacional, para construir un desarrollo armónico integral de progreso y paz, para así vivir una pedagogía liberadora comprometida de todos los grupos sociales en una relación de 
convivencia. De esta manera se debe activar una transformación curricular de experiencias y saberes que vinculen en una categoría de capacitación de habilidades y destrezas en una valorización del trabajo productivo, para deslastrar una educación obsoleta, tradicional y anacrónica, con una nueva postura filosófica de la enseñanza.

En tal sentido, Varone (2005), menciona que "el docente deberá dejar de constituirse en un pasivo consumidor de innovaciones para transformarse en un creador del currículo, llevar adelante una tarea educativa basada en sus propias estrategias de enseñanza" (pág. 18), lo cual significa que es el docente el transformador del currículo a través de la preparación que obtenga, siempre que se le dé la oportunidad de participar ampliamente en la reformulación del mismo, y así reflejar de forma creativa, los cambios requeridos en función de su interacción con el medio y la acumulación de experiencias en su actividad de enseñanza.

La gestión docente tiene que ir encaminada en la construcción de una vitalidad teórico-práctica, con un fin radical. La misma tiene que ir vinculada al estado de derecho, al currículo del sistema Educativo Nacional Bolivariano, que sea el timón de dirigir el barco que es capitaneado de manera armónica en docente y el estudiante.

De igual forma la gestión docente, tiene que transitar el camino en la búsqueda de la verdad, de erradicar la exclusión del ciudadano en la educación venezolana, tomando en cuenta la política educativa nacional en que esta tiene que aproximarse a la realidad histórico social y económico del país, para así transformar el paradigma nacional venezolano, a ser humanista que forje a los niños y niñas y adolescente del mañana, actos para vivir en la convivencia de la democracia.

La sociedad requiere un cambio en la gestión de las políticas educativas para propiciar una fuente vigorosa y útil, para insertar la educación a los cambios que plantea el hombre venezolano de una meritocracia de calidad 
docente, para brindar una congruencia a los retos del cambio social de una sociedad que está en permanente movimiento en las teorías curriculares y de modelos educativos, que no sean una política meramente demagógicas de adoctrinamiento ideológico de las elites que ostentan el poder en el campo económico, político, social, cultural y religioso.

Se debe crear un diseño curricular discutido en todos los campos e instituciones de la sociedad contemporánea venezolana, para que en un consenso de dialogo de pactos y acuerdos en que los docentes participen, con un tratado filosófico, político y teológico llegar a un acuerdo, de una educación de excelencia para mancomunar todos los de una política educativa integral, interdisciplinariamente para el desarrollo y progreso integral de la sociedad venezolana.

Es de reseñar que a la hora de ejecutar transformaciones originadas dentro del contexto educativo, es necesario tomar en cuenta que existen muchos actores que pueden influir, una de ellas sería la resistencia al cambio dentro de la cuales pueden existir diferentes obstáculos: conflictos de valores, considerando que las instituciones y los profesores son portadores de supuestos e ideologías potencialmente contradictorias con la nueva propuesta, conflictos de poder entre los profesores, directivos y técnicos, poca capacitación, falta de recursos las condiciones de trabajo de los docentes y las tradiciones diversas incorporadas a las reglas de pensamiento y acción de los actores, la falta de motivación al docente para el cambio, por nombrar algunas.

Otro de los aspectos influyentes en la gestión docente de Educación Primaria en la aplicación de los nuevos diseños curriculares, es el poco conocimiento del educador en cuanto a los diseños curriculares, existe la necesidad de la actualización por parte del mismo.

Al respecto Pérez (2004a), afirma que cuando se habla de innovación educativa, esta supone una serie de transformaciones en todos los ámbitos, por ello sostiene que: Innovar no solo implica hacerlo desde el ambiente, sino 
transformar a las personas desde sus propias concepciones de aprender y de conocer, darse cuente que la innovación se da en las estructuras cognitivas y en la concepción que se tiene del aprendizaje en relación a los sujetos (...) "concebir a los sujetos que aprenden desde perspectivas distintas en el aprendizaje, permite innovar las prácticas educativas" (pág. 1).

Con relación a los señalamientos anteriores es necesario indicar que la pedagogía de la creatividad se impone, puesto que a cambio de la acción que tiene límites predeterminados y es conocida a priori, toda la educación es una aventura en el cual juegan todas las capacidades del ser y no únicamente las intelectivas. Para que exista un buen desempeño docente, el educador debe implementar estrategias adecuadas y actualizadas.

En las Orientaciones Pedagógicas 2015-2016, existen propuestas muy interesantes, que con unas buenas estrategias y un seguimiento a las actividades a desarrollar, se podrían lograr los objetivos propuestos, para una educación de calidad con un diseño curricular acorde a la realidad en que vive el país.

Es importante señalar que la calidad de los estudiantes en primaria y bachillerato ha desmejorado mucho en el sentido de ya no se repiten grados como anteriormente, desconociendo así los rasgos de rendimiento alcanzado, esto no quiere decir que si el estudiante no repite no es buen alumno, sino que hay que promoverlo sin haber alcanzado ni si quiera las competencias mínimas, para ser promovido al grado inmediato superior.

Es evidente que no ha existido un cambio notorio en educación, en las acciones implementadas desde el Ministerio del poder popular para la Educación en la llamada consulta Nacional por la calidad educativa, por la cual imponen un currículo Nacional Bolivariano para las escuelas los las de Educación Primaria, igualmente se publicó una colección de libros titulados como colección Bicentenaria, donde tratan de impactar ideológicamente a los estudiantes. 
De igual manera Palacios (1997), Explica que;

Está el problema de los métodos en lo relativo a la educación moral escuela nueva no impone tampoco nada en este terreno. Se limita a hacer posible la autonomía de los escolares y a facilitarles el aprendizaje de libertad. Según el nuevo espíritu, el ideal al que se debe tender es a que el niño se conduzca a sí mismo: tras un periodo más o menos largo de aprendizaje, todo niño debe llegar a dirigirse a sí mismo. Al igual que las ideas que el niño adquiere con su voluntad son estériles y muertas, mientras permanecen vivas las que han nacido de la curiosidad y el interés, solo son morales los hábitos que han adquirido no a través del adiestramiento, sino por la experiencia de la relación con los compañeros, por la reflexión personal sobre la vida y la acción propia. (pág. 78).

Lo cual quiere decir, que en esta educación, no se toma en cuenta los valores Morales, importantes para la vida en sociedad y que se deja al estudiante al libre albedrío, sin tener una orientación del docente, dejándolos que se guíen por su propia voluntad, sin importar las consecuencias.

Por otro lado, hay que considerar la seguridad social de los docentes, su formación como lo establece la Ley Orgánica de Educación (2009), en su artículo 97 señala que el Ministerio de Educación... "establecerá para el docente programas permanentes de actualización de conocimientos, especialización y perfeccionamiento profesional" (pág. 27-28). Lo cual significa que el estado está obligado a brindar el perfeccionamiento adecuado a los profesionales de la docencia, con personal capacitado para ello y con un máximo de duración, no unos postgrados y Doctorados que solamente duran seis meses de adoctrinamiento ideológico.

Si se tomará en consideración que estamos en un mundo de la tecnología, en una sociedad globalizada, si el estado diera un seguimiento al material de dotación como las canaimitas, en las cuales ha hecho una inversión considerable sin obtener resultados satisfactorios, dichas canaimas se las lleva los estudiantes para sus hogares, sin darle el verdadero uso 
adecuado, las venden, les bajan vídeos inadecuados de pornografías y juegos sangrientos donde se manifiesta violencia.

\section{Cierre}

Según documento de la Cámara Venezolana de Educación Privada CAVEP (2014), en materia de calidad educativa para todos desde un enfoque de derechos humanos, destacan la importancia que tiene el pleno ejercicio de la educación, exige que esta sea de calidad.

Ofrecer una educación de calidad implica la articulación gobierno, educación, sociedad y familia, y se caracteriza por la transmisión de formas de actuar democrática, de fraternidad, basada en la pedagogía del aprendizaje significativo, donde se enseñe y se aprenda en y desde la libertad, que genere calidad de vida con visión de país a partir de un proyecto de Nación que es compartido por las mayorías (pág. 15).

Además de ello se requiere que el MPPE desarrolle políticas y formación docente adecuada a las nuevas realidades y con la flexibilidad necesaria para que las instituciones docentes desarrollen nuevos programas de formación, de igual manera dignificar la profesión del educador según Pérez (2004b), "invertir en la educación no solo implica el acceso de más niños y adolescentes al sistema educativo, no es suficiente con definir políticas, estrategias, con transformar los centros educativos y otorgarles más recursos económicos" (pág. 18).

Por ello invertir en educación implica también invertir tiempo para velar que el desempeño docente sea eficiente, en particular, en las labores que cotidianamente tiene que cumplir el docente en un aula de clases con sus estudiantes para su logro de desarrollo integral y así evitar el pragmatismo, el inmediatismo y la improvisación durante el desarrollo de las múltiples actividades estratégicas al conducir una sesión de clase.

A esto hay que añadir las funciones técnico-administrativas referidas al 
control y la supervisión de los procesos que dan fe de su situación, es decir, de todo el cúmulo de planes que debe concretar y que se efectúa antes del desarrollo de las actividades pedagógicas en el salón de clases. En este sentido, el desempeño docente cubre aspectos tales como: la planificación, ésta supone actividades como el diagnóstico de la situación de los educandos antes del inicio del primer lapso, la ambientación del aula, el perfil del egresado que se desea formar en cada año académico, la inscripción inicial con todos sus datos completos, el control de estadísticas, dotación de recursos, por nombrar algunos aspectos administrativos.

De igual manera va también implícita, la evaluación; la dirección y la supervisión de todos los docentes, a fin de determinar la efectividad del propio desempeño del educador, en su esfuerzo pedagógico, como la evaluación del alumno y la eficacia de los componentes del diseño curricular en función de los recursos disponibles en cada una de las instituciones.

Las más recientes concepciones de las funciones técnico-docentes parten del actuar como un facilitador del aprendizaje, como un promotor de experiencias educativas, con capacidad para utilizar estrategias y recursos que produzcan en el educando desarrollo de la creatividad, buena adquisición de conocimientos, habilidades y destrezas a situaciones de la vida real y el desarrollo de actitudes y valores.

Lo anterior se inscribe en un marco de principios éticos valores que caracterizan el ejercicio de la función docente. En consecuencia, el maestro es ético cuando actúa con responsabilidad en un contexto institucional, teniendo en cuenta que comparte con sus estudiantes tanto su ser como su hacer y el conocimiento que posee. La formación permanente se constituye en un elemento fundamental para asumir con compromiso y criterio la formación de sus alumnos, para ello requiere de proceso de actualización permanente.

El logro de un sistema educativo de calidad es un reto al que se enfrentan los sistemas educativos. El campo educativo en Venezuela siempre 
ha estado en crecimiento, con miras a buscar mejoras para lograr una educación de calidad.

\section{Referencias}

Botero, C. (2009a,b). Gestión Educativa. En Revista Iberoamericana de Educación. Edición: Organización de Estados Iberoamericanos para la Educación, la Ciencia y la Cultura (OEI). N49/2.

Carrasco, S. (2002). Gestión Educativa y Calidad de Formación Profesional en la Facultad de educación de la UNSACA. [Artículo en línea]. Recuperado de:

http://cybertesis.unmsm.edu.pe/xmlui/handle/cybertesis/1589

CAVEP (2014). La transformación del Sistema Educativo Venezolano, un

Reto Ineludible. [Artículo en línea]. Recuperado de:

http://www.cerpe.org.ve/tl files/Cerpe/contenido/documentos/Calidad \%20Educativa/Propuesta\%20Cavep\%20al\%20ME\%20(2).pdf

Díaz, F. \& Hernández G. (2001). Estrategias docentes para un aprendizaje significativo, una interpretación constructivista. México: Editorial McGraw-Hill.

Ministerio del Poder Popular para la Educación (2007). Currículo Nacional Bolivariano. Caracas, Venezuela: Edición: Fundación Centro Nacional para el mejoramiento de la Enseñanza de Ciencia, CENAMEC.

Ministerio del Poder Popular para la Educación (2009). Ley Orgánica de Educación. Recuperado de:

http://www.urbe.edu/portal-biblioteca/descargas/Ley-Organica\%20de-

Educacion.pdf

Montenegro, I. (2003). Evaluación del Desempeño Docente. Colombina Editorial Magisterio.

Orientaciones Pedagógicas (2014-2015). Instrucción Ministerial. Recuperado de: 
https://observatorioeducativo.files.wordpress.com/2015/01/orientacion es-pedagc3b3gicas-ac3b1o-escolar-2014-2015-lunes-15-sept-1.pdf Orientaciones Pedagógicas Año Escolar (2015-2016). Ministerio del Poder Popular para la Educación. [Artículo en línea]. Recuperado de:

http://www.cerpe.org.ve/tl files/Cerpe/contenido/documentos/Actualida d\%20Educativa/Formacion\%20Tecnica/Orientaciones\%20Pedagogica \%202015-2016\%20Carta-Min.pdf

Palacios, J. (1997). La Educación en el Siglo XX. (I) La tradición Renovadora. Editorial Laboratorio Educativo. Caracas, Venezuela.

Pérez, V. (2004a,b). ¿Cuándo aprenderemos a ser hermanos? El Márquez. Caracas, Venezuela: Ediciones Tripoides.

Ramírez, C. (2012). La gestión educativa (ge) en la educación básica y media oficial de manizales: un análisis desde las teorías administrativas y organizacionales. [Artículo en línea]. Recuperado de: http://www.bdigital.unal.edu.co/9039/1/7708531.2012.pdf

UNESCO (1996). La Educación Encierra un Tesoro. Informe a la UNESCO de la Comisión Internacional sobre la Educación para el Siglo XXI, presidida por Jacques Delors. Santillana, Ediciones UNESCO. [Artículo en línea]. Recuperado de:

http://www.unesco.org/education/pdf/DELORS S.PDF

Vargas, LL. (2008). Análisis de cinco desafíos en el ejercicio de la Administración Educativa. Revista electrónica Actualidades investigativas en Educación. ISSN: 1409-4703, enero/abril, año/Vol., 8, número 1, págs. 1-15. Universidad de Costa Rica. San José de Costa Rica. Recuperado de:

https://revistas.ucr.ac.cr/index.php/aie/article/download/9307/17769

Varone, F. (2005). Nueva gestión pública y participación Ciudadana. En Revista en políticas Sociales en Europa. Monográfico. La nueva gestión Pública. \#17. Madrid (España): Editorial Hacer. 


\section{Marilin Pastora Arrieche Cordero \\ e-mail: marinarrieche@hotmail.com}

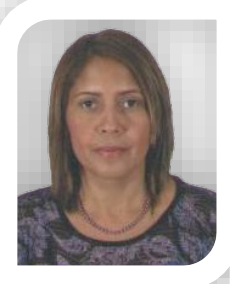

Nacida en Venezuela. Profesora en Educación Comercial (Universidad Pedagógica Experimental Libertador Barquisimeto), Magíster en Educación Técnica. (Universidad Pedagógica Experimental Libertador Barquisimeto). Diplomado en la Enseñanza del Inglés para la Escuela Primaria (Universidad Pedagógica Experimental Libertador Barquisimeto en convenio con el Consulado Británico). Estudiante de Doctorado en Ciencias de la Educación en la UFT. Docente titular activo del Ministerio del Poder Popular para la Educación en la escuela Bolivariana Jornada Completa "Ciudad de Maracaibo". Categoría docente V, subdirectora.

El contenido de este manuscrito se difunde bajo una Licencia de Creative Commons ReconocimientoNoComercial-Compartirlgual 4.0 Internacional 